\title{
Probing the surface of oxidized carbon nanotubes by selective interaction with target molecules
}

\author{
Rui Gusmão, ${ }^{a, b}$, Manuel Melle-Franco ${ }^{c}$, Dulce Geraldo ${ }^{b}$, Fátima Bento $^{*, b}$, Maria C. Paiva ${ }^{a}$, \\ Fernanda Proença ${ }^{b}$ \\ a) Instituto de Polímeros e Compósitos/I3N, Universidade do Minho, Campus de Azurém, 4800-058 \\ Guimarães, Portugal \\ b) Centro de Química, Universidade do Minho, Campus de Gualtar, 4710-057 Braga, Portugal \\ c) Centro ALGORITMI, Universidade do Minho, Campus de Gualtar, 4710-057 Braga, Portugal
}

*fbento@quimica.uminho.pt

\begin{abstract}
:
Screen printed carbon electrodes (SPCE) were modified with pristine and oxidized carbon nanotubes (CNT $T_{\text {prist }}$ and $\mathrm{CNT}_{\text {ox }}$, respectively). The extent of CNT oxidation evaluated by TGA increased with the time of reflux in nitric acid. The $\mathrm{CNT}_{\text {ox }}$ surface coverage by quinoid/phenolic functional groups was quantified by their voltammetric response at low redox potentials. Cyclic voltammetry was performed with SPCE-CNT $T_{\text {ox }}$ on solutions of hydroquinone, dopamine and ferricyanide used as model compounds. The sensitivity of reduced/oxidized forms of these molecules to the oxygen-containing functional groups of the CNTox was evaluated by the current intensity. Compounds with phenol/amine groups show a large increase of the current intensity with increasing CNT oxidation, while for carbonyl containing compounds only a negligible effect was perceived. Molecules that can hydrogen bond to the CNT functional groups interact better with the electrode surface, and this is supported by theoretical calculations. This showcases the use of CNT ox modified SPCE for selective sensing.
\end{abstract}

Keywords: Carbon nanotubes oxidation; Screen-printed carbon electrodes; Cyclic voltammetry; Dopamine; Hydroquinone. 


\section{Introduction}

The modification of electrodes with carbon nanotubes (CNT) is currently employed in numerous applications as a way to improve their performance. The presence of metallic ions, nanographitic and amorphous carbon impurities in pristine CNT is recognized to be in the origin of CNT electrocatalytic properties. Compton's group has shown that trace amounts of metal impurities play a critical role in the electrocatalytic properties of CNT [1]. Likewise, Pumera's group demonstrated that the electrocatalytic oxidation of amino acids by CNT was actually induced by the presence of metal-based impurities [2]. The nanographitic and amorphous carbon impurities on CNT were also found to facilitate the heterogeneous electron transfer $(\mathrm{HET})$ to biological compounds $[3,4]$. Since the nature and amount of impurities may vary markedly, comparison of results using CNT from different sources can be extremely challenging and their purification is generally performed before use.

There are different purification methods for CNT [5], based on the use of surfactants, organic solvents or oxidation by acids. The CNT treatment with concentrated solutions of mineral acids [6] can be carried out by reflux, sonication or microwave treatment. These procedures can last from a few minutes to several hours [7]. The acidic treatments can be effective in removing metal ions trapped between the CNT walls [5]. Oxidation also opens holes in CNT walls and cut the tubes [5]. The creation of edge-plane defect sites in the CNT $\mathrm{sp}^{2}$ structure and introduction of oxygen-containing moieties [6] are identified as key factors that may favor [5,8,9] or be detrimental [10] to specific electrochemical reactions.

Although it is recognized that the performance of CNT-modified electrodes is affected by their oxidation, the selective properties of electrodes modified by oxidized CNT are not yet clear. The effect of oxidation extent on the nature of oxygen functional groups is not thoroughly understood and all contributions to clarify this matter are relevant.

The work reported herein explores the effect of the oxidation extent of multiwall CNT by reflux with concentrated nitric acid during different times. The functionalization degree of oxidized CNT (CNT ox $)$ is characterized by thermogravimetric analysis (TGA). The voltammetric performance of screen-printed carbon electrodes modified with oxidized CNT (SPCEs-CNT ox ) is evaluated using relevant analytes, such as dopamine and hydroquinone and is correlated with the surface coverage by oxygen groups. The nature of oxygen containing moieties developed by the oxidative treatment is discussed considering the voltammetric results.

\section{Experimental Section}

CNT oxidation. Pristine multiwall CNT (CNT prist, Nanocyl NC 7000) were mixed with $100 \mathrm{~mL}$ of $65 \%$ $\mathrm{HNO}_{3}$ and refluxed for 1-8 hours at $110^{\circ} \mathrm{C}$. Samples were cooled down and the dispersion was centrifuged for $20 \mathrm{~min}$ at $6000 \mathrm{rpm}$. The sedimented $\mathrm{CNT}_{\text {ox }}$ were filtered through a polycarbonate filter 
(Whatman pore size $0.45 \mu \mathrm{m}$ ). Subsequently, $\mathrm{CNT}_{\text {ox }}$ were rinsed with water until the filtrate reached a neutral $\mathrm{pH}$. $\mathrm{CNT}_{\text {ox }}$ were dried in vacuum overnight at $80^{\circ} \mathrm{C}$ (Büchi glass oven $\mathrm{B}-580$ ).

Electrodes. SPCEs (DropSens DRP-110) with a carbon disk working electrode $(\mathrm{r}=2 \mathrm{~mm}$ ), a carbon auxiliary electrode and a silver pseudo-reference electrode were used.

SPCEs modification. SPCEs were modified by depositing $4.7 \mu \mathrm{g}$ of CNT by drop casting of $\mathrm{CNT}_{\text {ox }}$ suspensions (1.12 $\pm 0.06 \mathrm{mg} / \mathrm{mL}$, ethanol: water (1:1)) or $\mathrm{CNT}_{\text {prist }}$ suspensions $(0.60 \pm 0.01 \mathrm{mg} / \mathrm{mL}$, methanol: water (1:1)) on the working electrode. The drop was then left to dry in air.

Voltammetry. Cyclic voltammetry was carried out by placing $50 \mu \mathrm{L}$ of solution to cover the three electrodes. Measurements were performed with an Autolab PGSTAT30 potentiostat/galvanostat (Ecochemie), controlled by GPES software. Solutions of dopamine (DA; Sigma) and hydroquinone ( $\mathrm{HQ}$, May \& Baker, Ltd.) were prepared in $0.15 \mathrm{M}$, pH 3.2 or 7.4 , phosphate buffers (PB), whereas potassium ferricyanide (FC, José Gomes Santos) solutions were prepared in $0.10 \mathrm{M} \mathrm{KCl}$.

TGA. A Modulated Thermogravimetric Analyser Q500 (TA Instruments) was used in gradient mode for analysis of the weight yield of CNT oxidation. Samples were heated from room temperature to 800 ${ }^{\circ} \mathrm{C}$ at $10^{\circ} \mathrm{C} \mathrm{min}^{-1}$ under a constant nitrogen flow of $60 \mathrm{~mL} \mathrm{~min}{ }^{-1}$.

\section{Results and Discussion}

Concentrated oxidizing acids are often used to eliminate impurities present in CNT, but are also known to induce CNT oxidation [1,11,12]. The final oxidation level greatly depends on the oxidizing acid used, as well as on the reaction conditions (concentration, temperature and time). Therefore it is difficult to predict the extent to which CNT acid treatment can actually change the performance of CNT modified electrodes [13]. In order to understand this issue, we oxidized CNT by refluxing with concentrated nitric acid for different times (1, 2, 3, 6 and 8 hours). The $\mathrm{CNT}_{\text {ox }}$ were characterized by TGA and the response of modified electrodes was analyzed by cyclic voltammetry.

Figure 1a shows the weight loss of CNT due to thermal degradation, evaluated by TGA. CNT prist display a negligible weight loss at $800{ }^{\circ} \mathrm{C}(\approx 1 \%)$, in contrast, $\mathrm{CNT}_{\text {ox }}$ show increasing weight losses (from 8 to $23 \%$ ) with oxidation time, Figure 1a, inset. The weight loss provides an estimate of the functionalization degree as it results from the loss of chemical groups / molecules bonded to the CNT walls. Different functional groups can be introduced by oxidation such as: hydroxyl-phenolic, carbonylquinone, carboxyl and lactone $[6,14-16]$. The fact that the different weight vs. temperature curves of $\mathrm{CNT}_{\text {ox }}$ display similar shapes hints that the nature of the functionalization introduced by oxidation is similar despite the treatment duration.

Electrochemical characterization of the functional groups bonded to the CNT walls is shown in Figure 1b. Cyclic voltamograms (CVs) were obtained from SPCEs modified with $C N T_{\text {prist }}$ (SPCE-CNT prist) or with $\mathrm{CNT}_{\text {ox }}\left(\mathrm{SPCE}-\mathrm{CNT}_{\text {ox }}\right.$ ) by drop casting. While CVs from SPCE-CNT $T_{\text {prist }}$ exhibit a current that is 
mainly constant, those from SPCE-CNT ox display two broad bands corresponding to the oxidation and reduction of the $\mathrm{CNT}_{\text {ox }}$ functional groups. The small increase in capacitive currents observed for higher oxidation times may be due to an increase of the double-layer charge separation associated to a decrease of pi-pi interactions between CNTox and the electrode surface. In contrast, the faradaic current, which depends on the concentration of electroactive groups, increased with the $\mathrm{CNT}_{\mathrm{ox}}$ oxidation time, as depicted in Figure 1b.

Although the oxidation of CNT by $\mathrm{HNO}_{3}$ may introduce different types of functional groups, the voltammetric detection is only possible for those groups that can be oxidized or reduced in the accessible range of potentials of the SPCE $(-0.6$ to $0.6 \mathrm{~V})$. Within this range, the redox processes in $\mathrm{CVs}$ are associated with quinoid/phenolic groups, which are reduced or oxidized at potentials close to $0 \mathrm{~V}$ that depend on the local environment of the carbonyl/hydroxyl groups [16]. The presence of quinoid/phenolic groups (with a redox mechanism involving $1 \mathrm{H}^{+} / 1 \mathrm{e}^{-}$) is also supported by a shift of the voltammetric band of $-58 \mathrm{mV} / \mathrm{pH}$ unit (between $\mathrm{pH} 3.2$ and 7.4 , not shown). This value is close to the potential shift predicted by the Nernst equation [17].

The surface coverage, $\Gamma$, of the SPCE-CNT ox by quinoid/phenolic groups was estimated by the anodic charge, $Q$, (obtained by integration of the voltammetric band between -0.4 and $0.1 \mathrm{~V}$, Figure $1 \mathrm{~b}$ ) [1719] according to equation 1 :

The effective area, $A$, of the SPCE-CNT ox was estimated using Randle-Sevcik equation ${ }^{*}$ for a reversible electrochemical process under a semi-infinite planar diffusion control, from $\mathrm{CVs}$ recorded in $1.00 \mathrm{mM}$ solution of ferricyanide ( $F C, D=7.35 \times 10^{-6} \mathrm{~cm}^{2} \mathrm{~s}^{-1}$ ) [22] from 10 to $500 \mathrm{mV} \mathrm{s}^{-1}$ (not shown). The total TGA weight loss gradually increased with the oxidation time following a non-linear trend. A similar trend was observed for the $\Gamma$ values from the electroactive quinoid/phenolic groups. The linear correlation between $\Gamma$ and weight loss indicates that the formation of the electroactive groups progresses at the same rate as the overall CNT oxidation (Figure 1c).

Characterization of $\mathrm{CNT}_{\text {ox }}$ as electrode material was performed using three compounds: $\mathrm{FC}$, hydroquinone $(\mathrm{HQ})$ and dopamine (DA). The oxidation potentials of these species are higher than those of $\mathrm{CNT}_{\text {ox }}$ electroactive quinoid/phenolic groups. Therefore the oxidation of $\mathrm{FC}, \mathrm{HQ}$ and DA and reduction of their oxidized forms, occur at a surface containing quinoid rather than phenolic groups.

FC has a straightforward redox behavior with a fast HET that is known to be sensitive to electrode surface conditions $[21,23]$. CVs of FC using SPCE (SPCE-CNT prist $_{\text {or SPCE-CNT }}$ ox,8h) are shown in Figure 2a. For SPCE a peak-to-peak separation of $300 \mathrm{mV}$ is observed, whereas a smaller peak separation $(68 \mathrm{mV})$ is attained for SPCE-CNT prist $\left(100 \mathrm{mV} \mathrm{s}^{-1}\right)$, indicating that HET is favored by the CNT presence. Furthermore, the CNT oxidation treatment that is thought to affect both their surface

\footnotetext{
"This equation validity is limited by thin-layer effects associated to the porosity of the CNT layer $[20,21]$.
} 
structure and composition did not show any significant effect on the HET rate, as peak potentials are approximately unchanged. However, the oxidation treatment induced an increment in the voltammetric current which is probably linked to an increase in the concentration of CNT edges due to CNT breakage $[24,25]$. In comparison, a much larger increase in current intensity was found for $\mathrm{HQ}$ (Figure 2b) and DA (Figure 2c) These results are in accordance with literature where it is shown that surface oxides at carbon electrodes can greatly affect the electrode kinetics of quinones and dopamine $[26,27]$. For DA, both anodic and cathodic currents vary with CNT oxidation time, while for $\mathrm{HQ}$ a considerable anodic current variation and a marginal effect on cathodic current are observed. Remarkably, the anodic peak currents $\left(l_{\mathrm{ap}}\right)$ of $\mathrm{DA}$ and $\mathrm{HQ}$ vary linearly with $\Gamma$ while that of $\mathrm{FC}$ remains approximately constant (Figure 2d). Simultaneously, the cathodic peak current $\left(I_{\mathrm{cp}}\right)$ of DA shows a linear trend with $\Gamma$ whereas that of $\mathrm{HQ}$ is approximately unchanged. The lower sensitivity to SPCE$\mathrm{CNT}_{\text {ox }}$ oxidation degree by $p$-quinone ( $p-\mathrm{Q}$, the oxidized form of $\mathrm{HQ}$ ) may be due to a lower affinity of this molecule to the oxygen-containing functional groups of $\mathrm{CNT}_{\text {ox }}$, as compared with $\mathrm{HQ}$ and with the oxidized and reduced forms of DA. This observation is consistent with the quinoid nature of the oxygen groups bonded to the $\mathrm{CNT}_{\text {ox }}$ that may only form hydrogen bonds with the hydroxyl groups of $H Q$ and DA and with the amine group of DA.

Computer models were used to quantify the interaction of oxidized and reduced quinones with carbonyl functionalized graphenic edges modelled by coronene molecules. All calculations were performed with a home version of the program TINKER [28] using the MM3 force field. MM3 explicitly accounts for hydrogen bonds interactions and is one of the most accurate force fields for graphitic materials [29].

Three different molecules in oxidized and reduced forms were studied: $p-Q$, o-quinone $(o-Q)$ and $D A$. $O-Q$ was not studied experimentally and is an isomer of $p-Q$ directly related to DA. Coronene was functionalized with one and two neighboring carbonyl groups to give three different edge oxidations, Table 1. In order to approximate the global minimum simulated annealing, simulations were run for 1 nanosecond generating 200 snapshots/conformations for each adsorbate-substrate pair. These conformers were subsequently minimized and ranked in energy. Table 1 shows the results for the complexes with larger (more stable) intermolecular binding energy. The lowest relative binding energies, zero $\mathrm{kcal} / \mathrm{mol}$, are for the quinone molecules adsorbed, as in these systems hydrogen bonds cannot be formed. In the oxidized form of the DA molecule the amine group forms hydrogen bonds with the carbonyls of the substrate. These hydrogen bonds are weaker than for hydroxyl groups so this compound shows increased binding energies, $3-4 \mathrm{kcal} / \mathrm{mol}$ with respect to $o-Q$ and $p-Q$ but still lower binding energies than DA. Summing up, the reduced adsorbates show an increased binding energy $(5-9 \mathrm{kcal} / \mathrm{mol})$ than its oxidized counterparts and consequently are expected to have a larger affinity for carbonyl-quinone groups in $\mathrm{CNT}_{\text {ox }}$ matching the experimental measurements. 
Table 1 - Relative intermolecular binding energies in $\mathrm{kcal} / \mathrm{mol}$, for quinones and its reduced forms on carbonylfunctionalized coronene. Larger values indicate stronger binding energy. Absolute values can be obtained by adding $9 \mathrm{kcal} / \mathrm{mol}$.<smiles>O=C1C=CC(=O)C=C1</smiles>

$p-Q \quad H Q$<smiles>O=C1C=CC=CC1=O</smiles>

$O-Q$<smiles>OC1C=CC=CC1O</smiles>

Catechol<smiles>NCCC1=CC(=O)C(=O)C=C1</smiles>

Oxidized<smiles>NCCc1ccc(Cl)c(O)c1</smiles>

Reduced

DA

DA

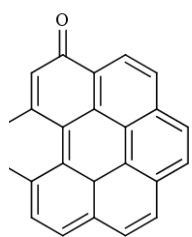

0
8

0

9

0

7

6

8

8
0
3

8<smiles>C=c1c(C)c2c3c4c5c(ccc6ccc7ccc1c(c24)c7c65)C=CC=3C</smiles>
Conclusions

CNT treated with nitric acid develop quinoid/phenolic groups which concentration increases with CNT oxidation time. $\mathrm{CNT}_{\text {ox }}$ display catalytic properties for HET similar to $\mathrm{CNT}_{\text {prist }}$ yet show a large sensitivity to $\mathrm{DA}$ and $\mathrm{HQ}$ due to favored binding through hydrogen bonding. The use of $\mathrm{CNT}_{\text {ox }}$ leads to increased current responses that can therefore be useful to improve selectivity and sensitivity of sensors.

\section{Acknowledgements}

Thanks are due to FCT and COMPETE-QREN-EU for financial support to the Research Centre CQ/UM, project scope UID/CEC/00319/2013 for financial support for the Centro ALGORITMI, and project PEst-C/CTM/LA0025/2013 (Strategic Project - LA 25 - 2013-2014) for financial support of 
IPC/I3N. RG thanks to FCT, POPH and ESF for his PostDoc Grant (SFRH/BPD/86690/2012). MMF acknowledges support by the FCT through the program Ciência 2008.

\section{References}

[1] G.G. Wildgoose, C.E. Banks, H.C. Leventis, R.G. Compton, Chemically Modified Carbon Nanotubes for Use in Electroanalysis, Microchim. Acta. 152 (2005) 187-214. doi:10.1007/s00604-005-0449-x.

[2] M. Pumera, Voltammetry of carbon nanotubes and graphenes: Excitement, disappointment, and reality, Chem. Rec. 12 (2012) 201-213. doi:10.1002/tcr.201100027.

[3] L. Wang, A. Ambrosi, M. Pumera, Carbonaceous impurities in carbon nanotubes are responsible for accelerated electrochemistry of acetaminophen, Electrochem. Commun. 26 (2013) 71-73. doi:10.1016/j.elecom.2012.10.018.

[4] X. Ma, L. Jia, L. Zhang, L. Zhu, The significant role of carboxylated carbonaceous fragments in the electrochemistry of carbon nanotubes, Chem. - Eur. J. 20 (2014) 4072-4076. doi:10.1002/chem.201304311.

[5] P. Hou, C. Liu, H. Cheng, Purification of carbon nanotubes, Carbon N. Y. 46 (2008) 2003-2025. doi:10.1016/j.carbon.2008.09.009.

[6] Y.-T. Shieh, G.-L. Liu, H.-H. Wu, C.-C. Lee, Effects of polarity and pH on the solubility of acid-treated carbon nanotubes in different media, Carbon N. Y. 45 (2007) 1880-1890. doi:10.1016/j.carbon.2007.04.028.

[7] F. Valentini, S. Orlanducci, M.L. Terranova, A. Amine, G. Palleschi, Carbon nanotubes as electrode materials for the assembling of new electrochemical biosensors, Sensors Actuators B Chem. 100 (2004) 117-125. doi:10.1016/j.snb.2003.12.036.

[8] F. Valentini, A. Amine, S. Orlanducci, M.L. Terranova, G. Palleschi, Carbon nanotube purification: preparation and characterization of carbon nanotube paste electrodes., Anal. Chem. 75 (2003) 54135421. http://www.ncbi.nlm.nih.gov/pubmed/14710820.

[9] A. Ambrosi, M. Pumera, Amorphous Carbon Impurities Play an Active Role in Redox Processes of Carbon Nanotubes, J. Phys. Chem. C. 115 (2011) 25281-25284. doi:10.1021/jp209734t.

[10] P. Cañete-Rosales, V. Ortega, A. Álvarez-Lueje, S. Bollo, M. González, A. Ansón, et al., Influence of size and oxidative treatments of multi-walled carbon nanotubes on their electrocatalytic properties, Electrochim. Acta. 62 (2012) 163-171. doi:10.1016/j.electacta.2011.12.043.

[11] X. Ji, R.O. Kadara, J. Krussma, Q. Chen, C.E. Banks, Understanding the Physicoelectrochemical Properties of Carbon Nanotubes: Current State of the Art, Electroanalysis. 22 (2010) 7-19. doi:10.1002/elan.200900493.

[12] I.D.I.I.D. Rosca, F. Watari, M. Uo, T. Akasaka, Oxidation of multiwalled carbon nanotubes by nitric acid, Carbon N. Y. 43 (2005) 3124-3131. doi:10.1016/j.carbon.2005.06.019.

[13] M. Pumera, B. Šmíd, K. Veltruská, Influence of Nitric Acid Treatment of Carbon Nanotubes on Their Physico-Chemical Properties, J. Nanosci. Nanotechnol. 9 (2009) 2671-2676. doi:10.1166/jnn.2009.031.

[14] J. Yang, K. Jiao, T. Yang, A DNA electrochemical sensor prepared by electrodepositing zirconia on composite films of single-walled carbon nanotubes and poly(2,6-pyridinedicarboxylic acid), and its application to detection of the PAT gene fragment, Anal. Bioanal. Chem. 389 (2007) 913-921. doi:10.1007/s00216-007-1450-5.

[15] P. Sharma, V. Bhalla, S. Tuteja, M. Kukkar, C.R. Suri, Rapid extraction and quantitative detection of the herbicide diuron in surface water by a hapten-functionalized carbon nanotubes based electrochemical analyzer, Analyst. 137 (2012) 2495-2502. doi:10.1039/c2an16235k.

[16] Y. Xing, L. Li, C.C. Chusuei, R. V Hull, Sonochemical oxidation of multiwalled carbon nanotubes, Langmuir. 21 (2005) 4185-4190. doi:10.1021/la047268e.

[17] M. Lu, R.G. Compton, Voltammetric pH sensor based on an edge plane pyrolytic graphite electrode, Analyst. 139 (2014) 2397-2403. doi:10.1039/C4AN00147H.

[18] C.A. Thorogood, G.G. Wildgoose, A. Crossley, R.M.J. Jacobs, J.H. Jones, R.G. Compton, Differentiating between ortho- and para-Quinone Surface Groups on Graphite, Glassy Carbon, and Carbon Nanotubes 
Using Organic and Inorganic Voltammetric and X-ray Photoelectron Spectroscopy Labels, Chem. Mater. 19 (2007) 4964-4974. doi:10.1021/cm071412a.

[19] G.G. Wildgoose, P. Abiman, R.G. Compton, Characterising chemical functionality on carbon surfaces, J. Mater. Chem. 19 (2009) 4875-4886. doi:10.1039/B821027F.

[20] I. Streeter, G.G. Wildgoose, L. Shao, R.G. Compton, Cyclic voltammetry on electrode surfaces covered with porous layers: An analysis of electron transfer kinetics at single-walled carbon nanotube modified $\begin{array}{lllllll}\text { electrodes, } & \text { Sensors } & \text { Actuators } & \text { B } & \text { Chem. } & 133 & \text { (2008) }\end{array}$ doi:http://dx.doi.org/10.1016/j.snb.2008.03.015.

[21] E.O. Barnes, X. Chen, P. Li, R.G. Compton, Voltammetry at porous electrodes: A theoretical study, J. Electroanal. Chem. 720-721 (2014) 92-100. doi:http://dx.doi.org/10.1016/j.jelechem.2014.03.028.

[22] S.J. Konopka, B. McDuffie, Diffusion coefficients of ferri- and ferrocyanide ions in aqueous media, using twin-electrode thin-layer electrochemistry, Anal. Chem. 42 (1970) 1741-1746. doi:10.1021/ac50160a042.

[23] R.L. McCreery, Advanced carbon electrode materials for molecular electrochemistry, Chem. Rev. 108 (2008) 2646-2687. doi:10.1021/cr068076m.

[24] M.N. Tchoul, W.T. Ford, G. Lolli, D.E. Resasco, S. Arepalli, Effect of Mild Nitric Acid Oxidation on Dispersability, Size, and Structure of Single-Walled Carbon Nanotubes, Chem. Mater. 19 (2007) 57655772. doi:10.1021/cm071758I.

[25] T.S. Miller, N. Ebejer, A.G. Güell, J. V Macpherson, P.R. Unwin, Electrochemistry at carbon nanotube forests: sidewalls and closed ends allow fast electron transfer., Chem. Commun.. 48 (2012) 7435-7437. doi:10.1039/c2cc32890a.

[26] R. Gusmão, V. López-Puente, I. Pastoriza-Santos, J. Pérez-Juste, M.F. Proença, F. Bento, D. Geraldo, M.C. Paiva, E. González-Romero, Enhanced electrochemical sensing of polyphenols by oxygenmediated surface, RSC Adv. 5 (2015) 5024-5031. doi:10.1039/C4RA12660B.

[27] R.L. McCreery, in: A.J. Bard (Ed.), Electroanalytical Chemistry, vol. 17, Dekker, New York, 1991.

[28] M. Melle-Franco, M. Prato, F. Zerbetto, Permanent chiral twisting of nonchiral carbon nanotubes, J. Phys. Chem. A. 106 (2002) 4795-4797. doi:10.1021/jp025657g.

[29] K. Strutyński, J.A.N.F. Gomes, M. Melle-Franco, Accuracy of dispersion interactions in semiempirical and molecular mechanics models: The benzene dimer case, J. Phys. Chem. A. 118 (2014) 9561-9567. doi:10.1021/jp506860t. 


\section{Figure captions}

Figure 1 - (a) TGA heating curves of $\mathrm{CNT}_{\text {prist }}$ (black) and $\mathrm{CNT}_{\text {ox }}$ at increasing oxidation times (1 to $8 \mathrm{~h}$ ). Inset: total weight loss at $800^{\circ} \mathrm{C}$. (b) $\mathrm{CVs}\left(100 \mathrm{mV} \mathrm{s}^{-1}\right)$ of SPCE-CNT prist and of CNT ox at increasing oxidation times (1 to $8 \mathrm{~h}$ ) in $0.15 \mathrm{M}$ PB solutions, $\mathrm{pH} 3.2$. (c) Relation between surface coverage $(\Gamma)$ and the weight loss at $800{ }^{\circ} \mathrm{C}$ $\left(y=0.12 x-0.16 ; R^{2}=0.997\right)$.

Figure $2-\mathrm{CVs}$ at $100 \mathrm{mV} \mathrm{s}^{-1}$ of SPCE and corrected CVs (blank subtracted) of SPCE-CNT prist and of CNT from: (a) $1.0 \mathrm{mM} \mathrm{FC}$ in $0.1 \mathrm{M} \mathrm{KCl}$, (b) $0.50 \mathrm{mM} \mathrm{HQ}$ and (c) $0.50 \mathrm{mM} \mathrm{DA}$ in $0.15 \mathrm{M} \mathrm{PB} \mathrm{pH} \mathrm{7.4.} \mathrm{(d)} \mathrm{Correlation}$ between the anodic peak intensities of DA $(\mathbf{\bullet}), \mathrm{HQ}(\boldsymbol{\square})$ and $\mathrm{FC}(\boldsymbol{\Delta})$ with the $\Gamma$ by oxygen groups. 

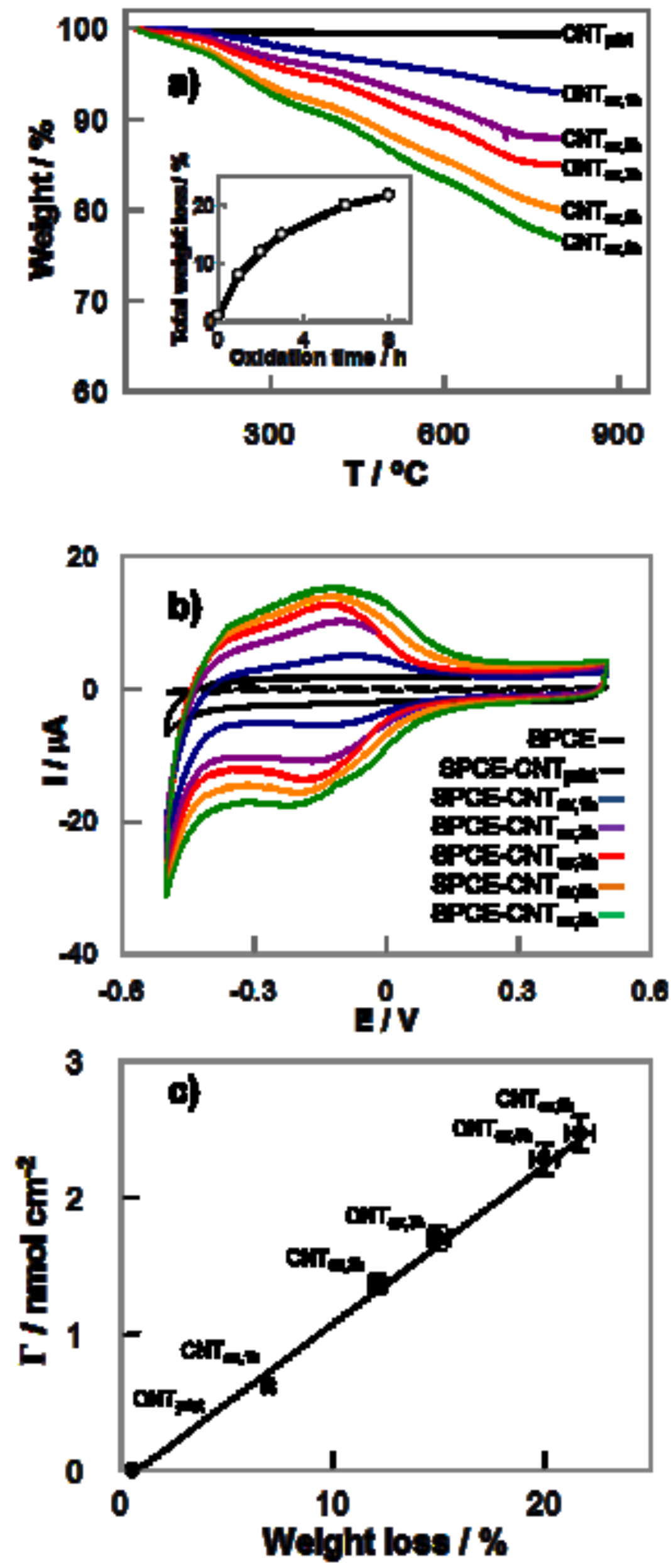

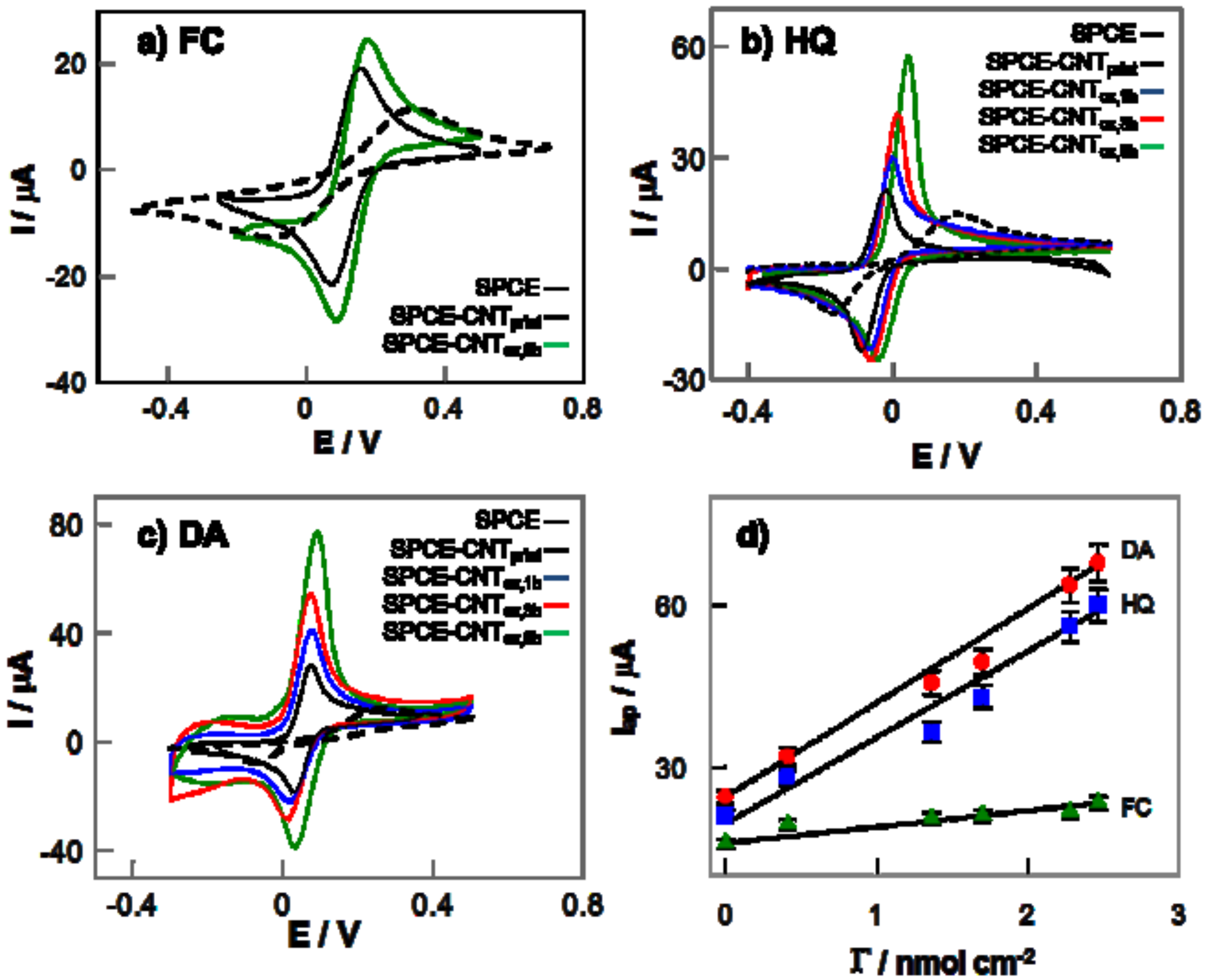